\title{
Pulmonary expression of the hepatocyte growth factor receptor c-Met shifts from medial to intimal layer after cavopulmonary anastomosis
}

\author{
Akio Ikai, MD \\ R. Kirk Riemer, PhD \\ Xiaoyuan $\mathrm{Ma}, \mathrm{MD}$ \\ Olaf Reinhartz, MD \\ Frank L. Hanley, MD \\ V. Mohan Reddy, MD
}

From the Department of Cardiothoracic Surgery, Division of Pediatric Cardiac Surgery, Stanford University, Palo Alto, Calif.

Read at the Eighty-third Annual Meeting of The American Association for Thoracic Surgery, Boston, Mass, May 4-7, 2003.

Received for publication May 2, 2003; revisions requested Aug 5, 2003; accepted for publication Sept 10, 2003.

Address for reprints: V. Mohan Reddy, MD, Department of Cardiothoracic Surgery, Division of Pediatric Cardiac Surgery, Stanford University, 300 Pasteur Dr, Falk CVRC, Palo Alto, CA 94305-5407 (E-mail: vmreddy@stanford.edu).

J Thorac Cardiovasc Surg 2004;127:1442-9

$0022-5223 / \$ 30.00$

Copyright (C) 2004 by The American Association for Thoracic Surgery

doi:10.1016/j.jtcvs.2003.09.009
Objective: Pulmonary arteriovenous malformations occur in up to $60 \%$ of patients after cavopulmonary anastomosis. We compared the effects of cavopulmonary anastomosis and pulmonary artery banding on lung gene expression in an ovine model to study the abnormal pulmonary vascular remodeling after the exclusion of inferior vena caval blood independent of reduced pulmonary blood flow. We previously demonstrated by contrast echocardiography that pulmonary arteriovenous malformations develop by 8 weeks after cavopulmonary anastomosis but not after pulmonary artery banding. Hepatocyte growth factor, a pleiotropic factor with morphogenic, mitogenic, and angiogenic activities, signals via its specific receptor c-Met to induce the antiapoptotic factor Bcl-2. In this study, we examined pulmonary artery expression of these factors and their potential role in pulmonary artery remodeling after cavopulmonary anastomosis and pulmonary artery banding.

Methods: Eighteen lambs aged 35 to 45 days were placed into 3 groups: cavopulmonary anastomosis, pulmonary artery banding, and control ( $n=6 /$ group $)$. In the cavopulmonary anastomosis group, the superior vena cava was anastomosed to the right pulmonary artery in an end-to-end fashion. In the pulmonary artery banding group, the left pulmonary artery was banded to reduce blood flow to $20 \%$ of control. The control group had a simple right pulmonary artery clamp for 30 minutes. Lung was harvested for Western blot, reverse transcriptase-polymerase chain reaction, and immunostaining at 2 weeks $(n=3 /$ group $)$ and 5 weeks $(n=3 /$ group $)$ after surgery.

Results: The expression of c-Met mRNA after cavopulmonary anastomosis was increased by twofold compared with the control or pulmonary artery banding group. The total lung expression of c-Met by Western blot was also up regulated at 2 weeks $(P<.05)$. However, total lung expression of hepatocyte growth factor and Bcl-2 by Western and reverse transcriptase-polymerase chain reaction was not different from the control and pulmonary artery banding groups at both 2 and 5 weeks after surgery. Immunohistochemical analysis revealed that c-Met expression was localized to the intimal layer of the pulmonary artery in the cavopulmonary anastomosis, while its expression in the control and pulmonary artery banding lungs was localized to the medial layer. Localization of Bcl-2 on the intimal layer in lambs with cavopulmonary anastomosis followed the same pattern as c-Met.

Conclusions: After cavopulmonary anastomosis, pulmonary artery expression of the hepatocyte growth factor receptor c-Met and one of its downstream effectors, Bcl-2, had increased in the intimal layer and decreased in the medial layer. Because the hepatocyte growth factor signaling promotes increased endothelial cell survival, it may have a role in pulmonary artery remodeling following cavopulmonary anastomosis. In addition, the change of c-Met expression in the medial layer after cavopulmonary anastomosis suggests a possible mechanism for the smooth muscle cell alteration related to abnormal angiogenesis. 


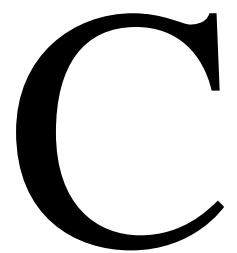

avopulmonary anastomosis (CPA) is performed in patients with single ventricle physiology as an interim procedure of total cavopulmonary connection., ${ }^{1,2}$ However, pulmonary arteriovenous malformations (PAVMs) develop in a significant proportion of patients undergoing CPA. Several series demonstrated clinically significant PAVMs in up to $25 \%$ of patients after $\mathrm{CPA}^{3,4}$ and asymptomatic PAVMs in up to $60 \%$ of patients. ${ }^{5}$ Clinical histological analysis of lung tissue after CPA reveals the increase of pulmonary microvessel density and dilated microvascular structures. ${ }^{6,7}$

However, the physiologic and molecular basis for this abnormal pulmonary vascular remodeling remains unclear. To better understand this phenomenon, we developed an ovine model that reliably produces pulmonary arteriovenous shunting 8 weeks after the construction of CPA. In general, the development of PAVMs after CPA suggests an abnormal form of angiogenesis. In our previous study, we reported the up-regulation of the pulmonary expression of vascular endothelial growth factor (VEGF) after not only a CPA model but also a pulmonary artery banding (PAB) model, which is a pulmonary blood flow-reducing model that does not result in the development of PAVMs. ${ }^{8}$

Therefore, we focused on an alternative angiogenic factor, hepatocyte growth factor (HGF) and its specific receptor c-Met, to determine its possible role in PAVM development following CPA. HGF is pleiotropic protein involved in mitogenesis, motogenesis, morphogenesis, and embryonic organogenesis. ${ }^{9}$ The expression of c-Met in the normal lung has not been reported so far. However, Ohmichi and colleagues ${ }^{10}$ reported that $\mathrm{HGF}$ acts a humoral mediator of mesenchyme-epithelial interaction during fetal lung development. Moreover, the expression of HGF in the lung has also been verified during liver regeneration after liver injury. ${ }^{11}$ In terms of the HGF system in the vascular structure, the expression of HGF and c-Met on the aortic endothelial and smooth muscle cells has been verified as a local autocrine/paracrine HGF system, which acts as an endothelialspecific growth factor without vascular smooth muscle cell replication. ${ }^{12} \mathrm{Bcl} 2$, which is an antiapoptotic factor and one of the downstream signals of activated c-Met, has been found to be up-regulated in a manner consistent with inhibition of the endothelial cell death in response to hypoxia. ${ }^{13}$

In this study, we evaluated the expression of the HGF system within the pulmonary artery following CPA and compared this with the control and PAB lambs, neither of which develop PAVM.

\section{Methods}

\section{Animal Model}

Western sheep, aged 35 to 45 days $(n=6)$, were anesthetized with isoflurane $(2 \%)$, intubated, and mechanically ventilated. After median sternotomy and pericardiotomy, the superior vena cava (SVC) and right pulmonary artery (PA) were identified and dissected free from their attachments. The right PA was divided near the pulmonary bifurcation and the proximal end was oversewn. The SVC was similarly divided at the cavoatrial junction and the atrial end was oversewn. Intravenous heparin was administered (300 U/kg), and $16 \mathrm{~F}$ to $20 \mathrm{~F}$ venous cannulas were placed to bypass the SVC to the right atrium. The SVC was then anastomosed to the right PA in an end-to-end fashion with running polypropylene sutures. The venous cannulas were removed, and the sternum was closed. After skin closure, the lambs were extubated and allowed to recover.

Another set of age-matched lambs underwent PAB $(n=6)$. After sternotomy, the left PA was dissected free from its attachments. The left PA was then encircled with an umbilical tape, and an ultrasonic flow probe (Transonic Systems, Inc, Ithaca, NY) was placed. Once baseline flow measurements were recorded, the umbilical tape was tightened around the left PA with surgical clips to reduce flow to $20 \%$ of baseline values. The band was then further secured by 5-0 polypropylene sutures and placed in the adventitia of the left PA.

A third group of lambs of the same age served as shamoperated control animals $(n=6)$. The preparation and dissection were the same as that described for the CPA cohort. The right PA was occluded with a vascular clamp for 30 minutes.

All of the animals in this study received humane care in compliance with the Principles of Laboratory Animal Care, formulated by the National Society of Medical Research, and the Guide for the Care and Use of Laboratory Animals, prepared by the Institute of Laboratory Animal Resources, National Research Council and published by the National Academy Press, revised 1996. The protocol was approved by the Committee on Animal Research at University California San Francisco and Stanford University.

\section{Tissue Harvest}

Lambs were killed at 2 and 5 weeks after the operation to obtain tissue for analysis. Animals were intubated and mechanically ventilated. After median sternotomy, the lambs were anticoagulated with sodium heparin $(300 \mathrm{U} / \mathrm{kg})$. The lambs were then killed by the infusion of sodium pentobarbital, the lungs were removed, and the samples were snap-frozen in liquid nitrogen. Tissue blocks were also fixed in HistoChoice media (Amresco Inc, Solon, Ohio) for histological analysis.

\section{Western Blot Analysis}

Specimens of the right lower lung tissue from the CPA and control cohort and the left lower lung tissue from the PAB cohort were obtained as described above. The specimens were homogenized, and the proteins were extracted as previously described. ${ }^{14}$ The proteins were then transferred to a polyvinylidene difluoride membrane (Amersham Pharmacia Biotech Inc, Piscataway, NJ). The membrane was soaked in Tris-buffered saline solution (100 $\mathrm{mmol} / \mathrm{L}$ Tris- $\mathrm{HCl}, \mathrm{pH} 7.5$ and $150 \mathrm{mmol} / \mathrm{L}, \mathrm{NaCl}$ ) containing 5\% nonfat powdered milk and $0.1 \%$ Tween-20 to block nonspecific binding. The membrane was then incubated overnight at $4^{\circ} \mathrm{C}$ with the following primary antisera (all from Santa Cruz Biotechnology, Santa Cruz, Calif) at the specified dilutions: c-Met, 1:100; HGF, 1:50; Bcl-2, 1:100. The blots were washed and incubated for 1 hour at room temperature with a peroxidase-conjugated second- 
ary antibody (1:30,000 dilution, Vector Laboratories, Inc, Burlingame, Calif). Immunoreactivity was detected with the Supersignal chemiluminescent substrate kit (Pierce Chemical Company, Rockford, Ill). Quantitative assessment of band densities was performed using a scanning densitometry (Quntity One quantitation software, Bio-Rad, Hercules, Calif).

\section{Reverse Transcriptase-Polymerase Chain Reaction}

Total RNA was prepared from homogenates of the right lower lobe lung tissue from the control and CPA lambs, as well as the left lower lobe lung tissue from the PAB studied at selected time points after the operations. RNA was extracted with the use of the TRI Reagent RNA isolation protocol (Molecular Research Center, Inc, Cincinnati, Ohio). Samples were incubated with oligo (dT) primer and reverse transcriptase (Superscript Kit; Gibco BRL, Life Technologies, Inc, Rockville, Md) at $42^{\circ} \mathrm{C}$ for 50 minutes. The resulting complementary DNA underwent polymerase chain reaction (PCR) with the use of the following primers: HGF forward primer 5'TGCACAATTCCTGAAAAGAC-3 and reverse primer 5'-CGGACAAAAATACCAGGACG-3' (product 314 bp), c-Met forward primer 5'-CGGTCTTCAAGTAGCCAAGG-3' and reverse primer 5'-ACCAGTTCAGAAAACGGATGG-3' (product $450 \mathrm{bp}$ ), and glyceraldehyde-3-phosphate dehydrogenase (G3PDH) forward primer 5'-TCCTTGAGGCCATGTGGGCCAT-3', reverse primer 5'-TGATGACATCAAGAAGGTGGTGAAG-3' (product $220 \mathrm{bp}$ ). PCR was initiated with 4 minutes of denaturation at $95^{\circ} \mathrm{C}$ followed by 30 cycles of 30 seconds of denaturation at $95^{\circ} \mathrm{C}, 30$ seconds of annealing at $55^{\circ} \mathrm{C}$, and 90 seconds of extension at $72^{\circ} \mathrm{C}$, followed by a final 7 additional minutes of extension (iCycler Thermal Cycler, Bio-Rad). PCR products were separated in a $1.5 \%$ agarose gel. Densitometric analysis was used to quantify the PCR signal (Quntity One quantitation software, Bio-Rad). Density data for each sample was normalized to its corresponding recovery control gene (G3PDH) density prior to comparison.

\section{Immunohistochemistry}

Immunolocalization was performed as previously described. ${ }^{7}$ In brief, lung tissue from the experimental and control animals at 2 and 5 weeks after $\mathrm{CPA}, \mathrm{PAB}$, or control surgery was fixed in HistoChoice media. Tissue was then paraffin embedded, sectioned at $7 \mu \mathrm{m}$, and mounted onto glass slides. Slides were deparaffinized by means of immersion for 3 minutes each in xylene and $100 \%$, $95 \%, 70 \%$, and $50 \%$ ethanol. Slides were washed with Trisbuffered saline solution (TBS; $50 \mathrm{mmol} / \mathrm{L}, \mathrm{pH} 7.6$ ) and then incubated with $0.5 \%$ hydrogen peroxide with $1 \mathrm{mg} / \mathrm{mL}$ of saponin in TBS for 30 minutes to quench endogenous peroxidases. Sections were incubated with $5 \%$ serum from the host species for the secondary antisera to reduce nonspecific antibody binding. Sections were also blocked using avidin and biotin blocking kit (Vector Laboratories) for 15 minutes. Sections were incubated for 18 hours at $4{ }^{\circ} \mathrm{C}$ with primary antisera as described above. Visualization of staining was accomplished with the Vectastain elite ABC kit (Vector Laboratories), with diaminobenzidine as the chromogen (DAB kit, DAKO, San Francisco, Calif).

\section{Statistical Analysis}

Data were analyzed with Statview (SAS Institute, Inc, Cary, NC). Protein and RNA analyses were evaluated with 3 animals at both
2 and 5 weeks in the CPA, PAB, and control groups for a total of 6 animals in each cohort. Analysis of statistical differences between groups at each time point was performed by means of nonparametric Mann-Whitney test. All values are expressed as mean \pm standard error mean (SEM).

\section{Results}

\section{HGF mRNA and Protein Expression After CPA and PAB}

We confirmed lung expression of HGF mRNA and protein in the lung. The HGF mRNA level in the CPA group did not reveal any differences as compared with the control and PAB groups at 2 weeks (Figure 1, A). The HGF $\alpha$-chain protein expression level in the CPA group was found to be the same as the control and PAB groups at 2 weeks and 5 weeks (Figure 1,B).

\section{c-Met mRNA and Protein Expression After CPA and PAB}

We demonstrated lung expression of c-Met mRNA and protein in our model. The expression of c-Met mRNA was increased 2.6-fold in the CPA group compared with the control group and 3.4-fold compared with the PAB group at 2 weeks $(P<.05$, respectively; Figure $2, A)$. c-Met protein expression was also increased 1.5-fold in the CPA group compared with the control and $\mathrm{PAB}$ groups at 2 weeks and 5 weeks (Figure 2, B). We also confirmed Bcl-2 protein expression in our lung model. However, we did not find any significant difference in Bcl-2 protein expression among the 3 groups.

\section{Localization of the HGF Signaling System in the Lung} HGF protein was found to be expressed in the smooth muscle layers of both vascular and airway tissues (Figure 3). c-Met protein was expressed in the epithelial cells and airway smooth muscle cells in all 3 groups. In the CPA lung, the PA expression of c-Met was predominantly observed in the endothelial layer at 2 weeks and 5 weeks, while its expression in the control and PAB lungs was predominantly observed in the medial layer (Figure 4). Bcl-2 expression in the CPA lung was localized to the intimal layer, consistent with the expression of c-Met. In contrast, Bcl-2 expression in the control and PAB lungs was mainly restricted to the medial layer of the PA (Figure 5).

\section{Discussion}

In the present study, we confirmed the existence of HGF and c-Met in pulmonary vessels. Under normal physiological conditions, c-Met is barely detectable in the lung. c-Met expression in the lung has been reported during fetal lung development as a mesenchyme-derived morphogenic factor. ${ }^{10}$ We used a growing lamb to simulate the clinical setting that PAVM after CPA has on younger patients who have higher incidence of this phenomenon. ${ }^{15}$ Our results 
A
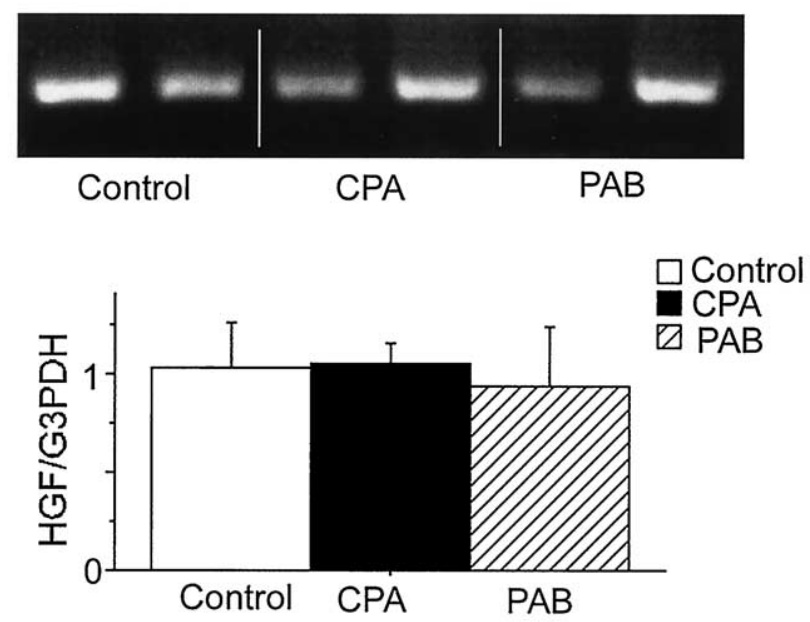

B
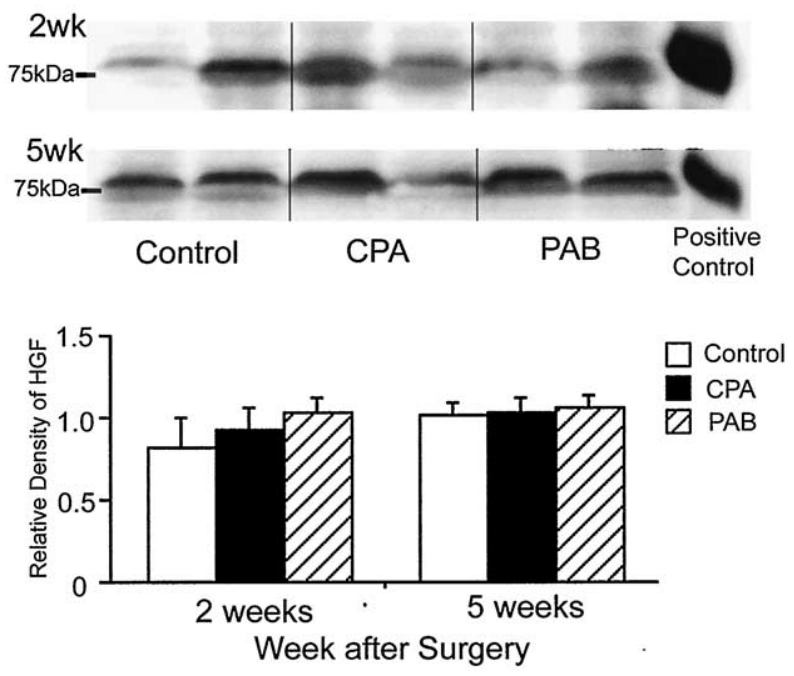

Figure 1. Expression of HGF mRNA and protein in the lung. A, Reverse transcriptase-polymerase chain reaction analysis of HGF mRNA isolated from the whole lung tissue 2 weeks after surgery. The upper image shows representative signal of 2 animals in each group. The lower image shows the result of quantitative analysis $(n=3)$. B, Western blot analysis of HGF $\alpha$ subunit. The upper image is a representative blot image of each group. The upper image is after 2 weeks and the lower image is after 5 weeks. The lower image is the result of quantitative analysis $(n=3)$. No statistically significant differences were observed.

suggested that HGF system might support organized lung development even after birth. We are unaware of any reports pertaining to the HGF system expression in the pulmonary vasculature in normal lungs. Therefore, we believe that this is the first report of c-Met expression in the pulmonary vasculature of a growing lung.
A
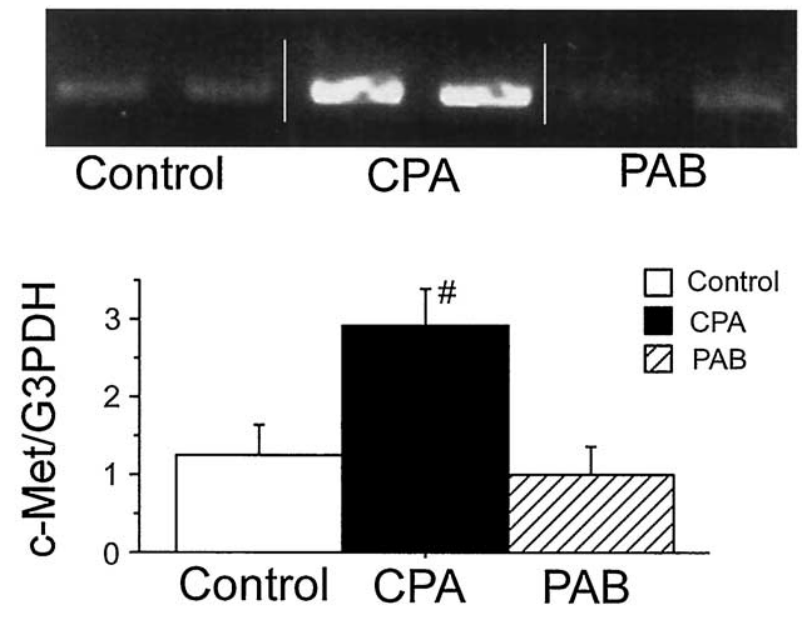

B
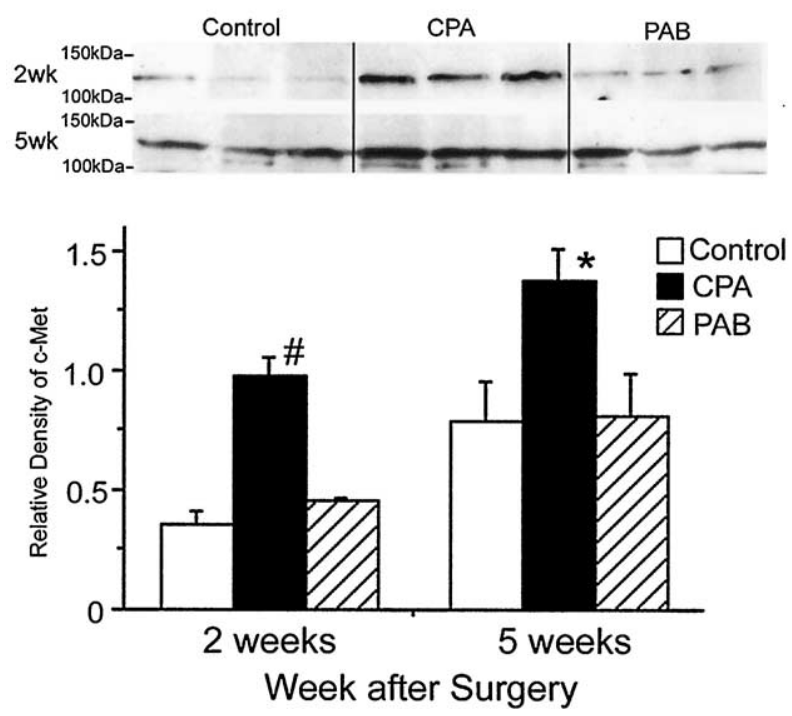

Figure 2. The expression of c-Met protein and mRNA in the lung. A, Reverse transcriptase-polymerase chain reaction analysis of c-Met mRNA, which has been isolated from the whole lung tissue 2 weeks after surgery. The upper image shows representative signal of 3 animals in each group. The lower lane is the result of quantitative analysis from the 3 animals per group. \#P $<.05$ versus control and PAB. B, Western blot analysis of $C-M e t$. The left 3 lanes are control lambs. The middle 3 lanes are CPA lambs. The right 3 lanes are PAB lambs. The upper image is after 2 weeks and the lower image is after 5 weeks. $\# P<.05$ versus control and PAB at 2 weeks. ${ }^{*} P<.05$ versus control and $P A B$ at 5 weeks.

We confirmed a similar pattern of HGF localization in the mesenchyme among the 3 groups by immunohistochemical stain. Liu and colleagues ${ }^{16}$ reported increased expression of c-Met and decreased expression of HGF in the 

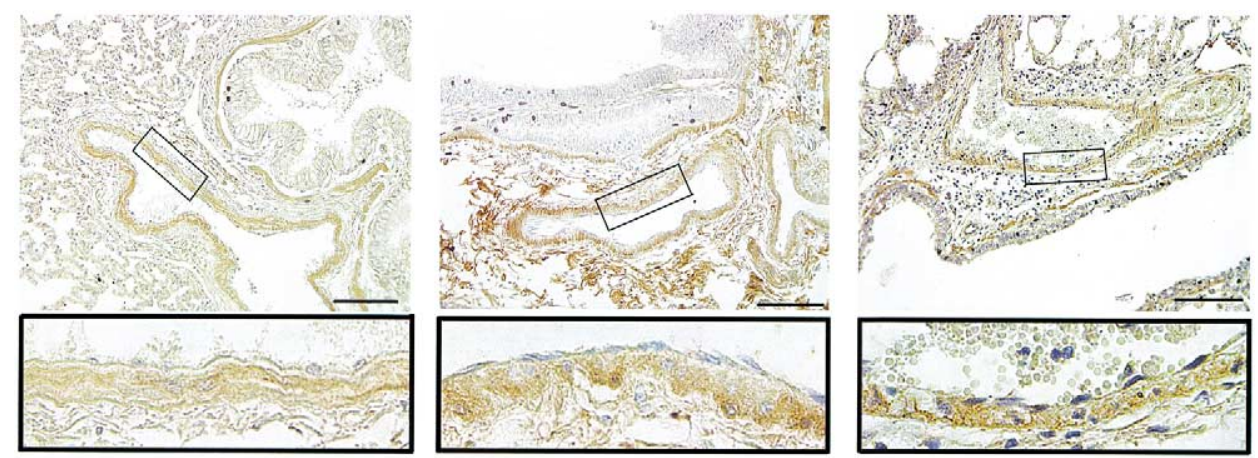

Figure 3. Immunohistochemical stain of HGF. The left image is control. The middle image is CPA. The right image is PAB. Original magnification is $200 \times$. Area enlarged in inset $(400 \times)$ is indicated by the black rectangle. The black bar indicates $100 \mu \mathrm{m}$.
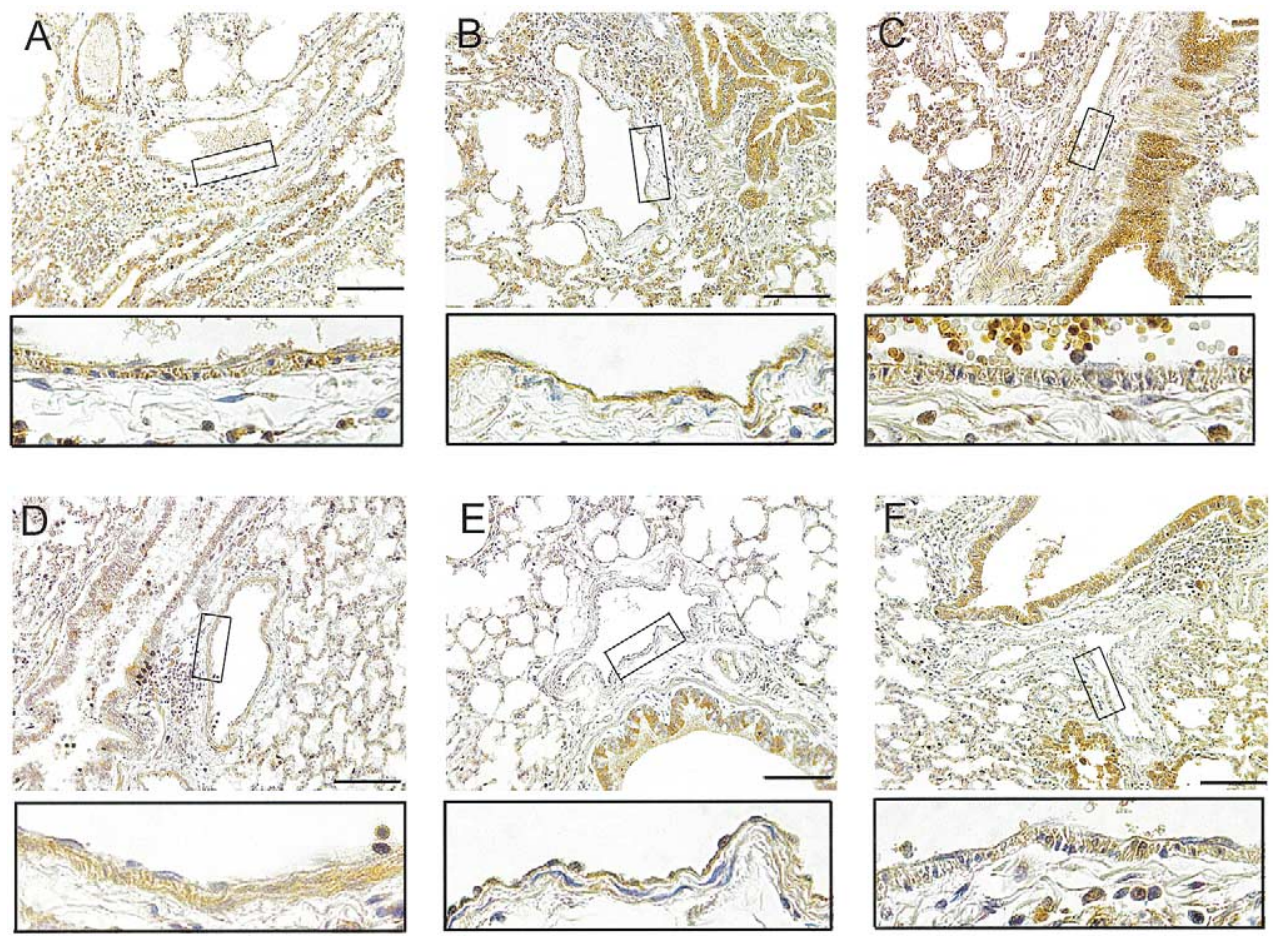

Figure 4. Immunohistochemical stain of c-Met. A, B, and C, after 2 weeks and D, E, and F, after 5 weeks. Both A and $D$ are control. Both $B$ and $E$ are CPA. Both $C$ and $F$ are PAB. The original magnification is $200 \times$. The area enlarged in inset $(400 \times)$ is indicated by the black rectangle. The black bar indicates $100 \mu \mathrm{m}$.

kidney after acute renal failure. They also indicated that circulating HGF up-regulates c-Met expression in the kidney after injury. Himeno and colleagues ${ }^{17}$ reported that HGF plasma concentration in the SVC is higher than in the hepatic vein. In reference to these facts, although we did not measure the plasma HGF level in this setting, we speculated that circulating HGF induces c-Met up-regulation in an endocrine manner. On the other hand, To and colleagues ${ }^{18}$ demonstrated that interleukin- 6 up-regulated the expression of c-Met in lung adenocarcinoma cells. These facts suggest that the induction of c-Met expression affects not only HGF/c-Met autocrine and paracrine mechanisms but also affects cytokine expression. We reported the up-regulation of oxidative stress genes in the CPA lung, ${ }^{8}$ which is consistent with the possibility that a stress-promoting factor could be present and active in the up-regulation of c-Met expression in the lung following CPA.

We documented that the expression of c-Met mRNA and protein level were up-regulated after CPA. In the CPA lung, moreover, we observed a consistent shift in the expression 

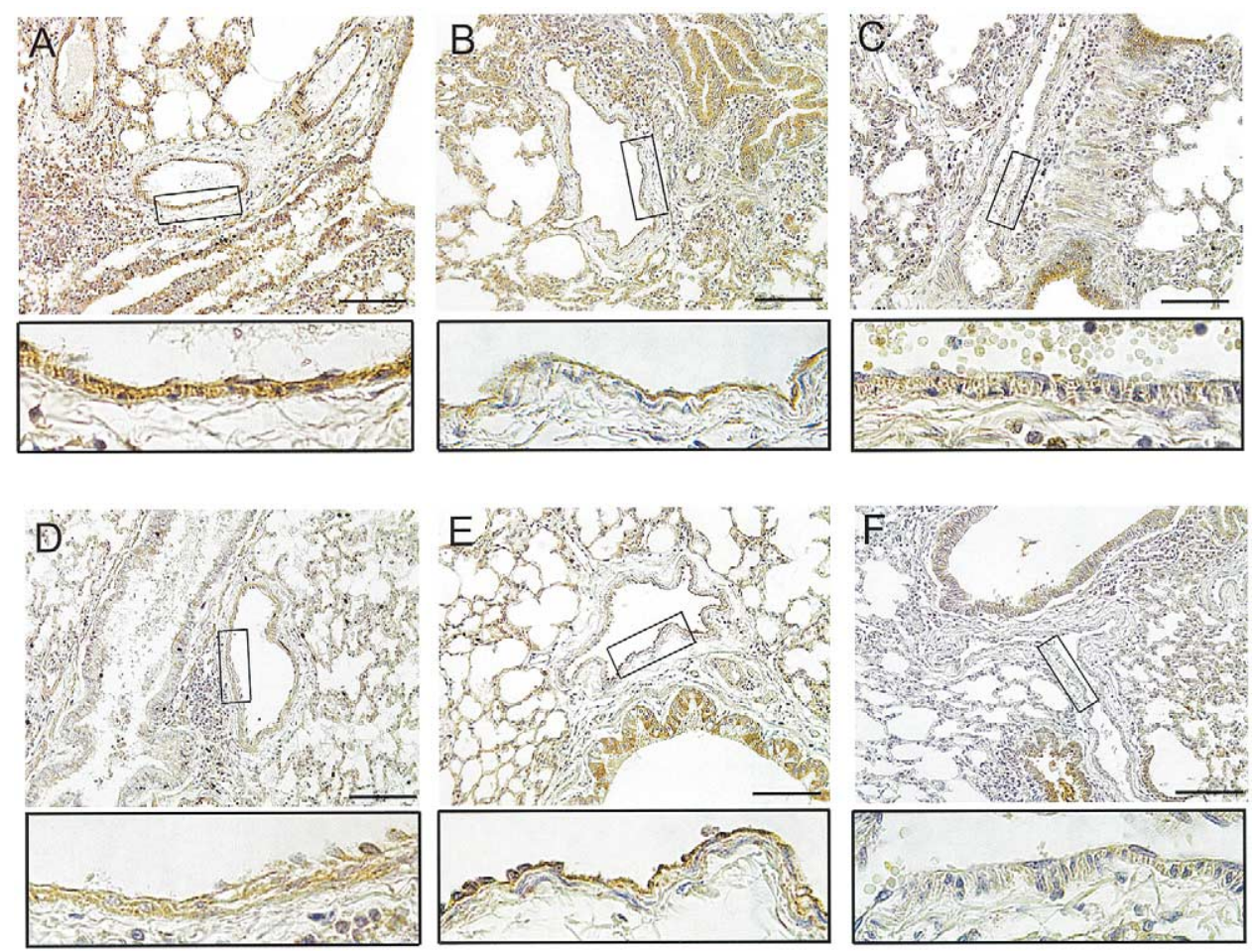

Figure 5. Immunohistochemical stain of Bcl-2. A, B, and C, after 2 weeks and D, E, and F, after 5 weeks. Both A and $D$ are control. Both $B$ and $E$ are CPA. Both $C$ and $F$ are PAB. The original magnification is $200 \times$. The area enlarged in inset $(400 \times)$ is indicated by the black rectangle. The black bar indicates $100 \mu \mathrm{m}$.

of c-Met and Bcl-2 from the medial layer to the intimal layer, while in the control and PAB lungs c-Met was expressed on the medial layer predominantly. Furthermore, HGF has been reported to exert an endothelial-specific effect to increase $\mathrm{Bcl}-2$ expression and attenuate hypoxiainduced endothelial cell death. ${ }^{13} \mathrm{Bcl}-2$ expression in the pulmonary endothelial cell in our model may therefore indicate activation of the c-Met signal transduction pathway, thus promoting pulmonary endothelial cell survival and supporting the development PAVM. Based on these findings, our results are consistent with the fact that c-Met up-regulation in the endothelial cell promotes angiogenesis in vitro. ${ }^{19}$ However, we were not able to clearly confirm significant differences in the protein expression of Bcl-2 among the 3 groups, as c-Met expression was increased. Because Bcl-2 is not a specific downstream signal for HGF system, its expression is likely to be affected by other signaling molecules. Haddad and colleagues ${ }^{20}$ demonstrated that Bcl-2 in the lung was decreased immediately after birth and restored at a later developmental phase. In our study, we speculate that $\mathrm{Bcl}-2$ expression in this model might have been affected by the developmental stages of this developing lamb. Furthermore, we believe that the primary importance of these signaling systems in the lung may not be the quantitative difference in expression but rather its differen- tial localization, which may be a more significant indicator of physiologic differences among the three groups.

We showed the existence of HGF on the medial layer among the 3 groups. Hayashi and colleagues ${ }^{21}$ reported that local HGF system works in a paracrine-autocrine manner between the endothelial cell and smooth muscle cell. HGF is also known to have motogenic and mitogenic activity on smooth muscle cells as well as endothelial cell. ${ }^{22} \mathrm{Bcl}-2$ expression in the smooth muscle cells in our control and PAB models might indicate the effect of HGF to act as a smooth muscle cell survival factor in an autocrine manner. One of the distinguishing features of HGF is that it stimulates the migration of vascular smooth muscle cells without their replication, whereas VEGF dose not stimulate either the migration or proliferation of vascular smooth muscle cell. ${ }^{23}$ In terms of the mechanism of the development of PAVM, our previous study documented that VEGF was actually up-regulated in of both the CPA and PAB models. ${ }^{8}$ In the present study, we propose that changes in the HGF signaling system in the PA, which are restricted to the setting of CPA, might be a more important differential factor in the development of PAVM than VEGF signaling system. The differential expression of c-Met among the $\mathrm{CPA}, \mathrm{PAB}$, and control groups suggests that the paracrineautocrine regulatory system of local HGF might be dis- 
rupted in response to, for example, oxidative stress or a lack of some substance derived from hepatic venous effluent. Interestingly, Ikai and colleagues ${ }^{24}$ reported disappearance of hypoxic pulmonary vasoconstriction after CPA, indicating abnormal property of the peripheral PA, especially in the smooth muscle cell, in response to alveolar hypoxia. Moreover, the abnormal smooth muscle cell with decreased expression of c-Met after CPA might be involved in altered angiogenesis, resulting in the development of PAVM by way of the HGF signaling system.

On the other hand, exclusion of inferior venocaval drainage from the pulmonary circulation could potentially interrupt the delivery of a mediator of normal vascular development that is derived from the liver or portal vein system. This theory is supported by reports of the resolution of PAVMs after the inclusion of inferior venous blood. ${ }^{25,26} \mathrm{In}$ hepatopulmonary syndrome, the improvement of PAVM after liver transplantation has also been reported similarly. ${ }^{27}$ Interestingly, HGF was originally discovered as a hepatocyte regeneration factor ${ }^{9}$ and the HGF local expression in the lung is up-regulated after partial hepatectomy. ${ }^{11}$ These observations suggest that the liver and lung participate in some endocrine cooperating system in response to the HGF and c-Met expression. As mentioned above, HGF is also related to lung development. ${ }^{10}$ Bernstein and colleagues ${ }^{15}$ reported that the prevalence of PAVMs was significantly higher in infants less than 6 months old. These facts suggest that lung development organized by the HGF signaling system may affect the development of PAVM. Further work is warranted to fully understand the mechanisms involved in formation of PAVMs after CPA.

There are limitations to our study. We studied the expression of HGF system only in the early postoperative phase. Our previous studies reported the development of PAVMs in the lung after CPA, which occurs within 8 weeks in all animal of our animal model. Some of the animals in our study, however, were positive for PAVM by contrast echocardiography within 5 weeks after CPA. Therefore, we chose the time points of 2 and 5 weeks after surgery to identify molecular changes that precede the development of PAVMs. In support of our approach, we showed consistent change of c-Met expression at 2 and 5 weeks Further study is needed to also fully evaluate the expression of the HGF system after the development of PAVMs.

In the present histological study, we evaluated the slightly larger-size PA compared with the PA that has been previously speculated to relate to PAVM development. However, the anatomical localization of PAVMs has not been described. From both of our previous and present study, we were not able to define a clear explanation on the pathological change in the development of PAVM in our lamb model. Although the change in the expression of c-Met in the large-size PA might not be direct evidence of the development of PAVM, any small vessels relating to PAVM should branch off the large PA. Further study is needed to characterize structural histopathology of PAVMs after CPA and the role of HGF signal transduction system in the development of these PAVMs.

We evaluated HGF signaling system in the lung of CPA lamb model in which development of PAVM was consistently reproducible. After CPA, the expression of c-Met, a specific receptor of HGF in the lung, increased. c-Met localization changed in different time points from the medial to intimal layer in the PA. Neither the control nor PAB group, however, exhibited any changes in the expression of c-Met. Bcl-2, an antiapoptotic factor, and one of c-Met's downstream signals showed similar localization as c-Met. These results suggest that endothelial cell survival promoted by HGF signaling system is a part of the mechanism responsible for the development of PAVM, and in turn, the change of c-Met expression in the medial layer after CPA suggests a possible mechanism for the smooth muscle cell alteration related to abnormal angiogenesis or PAVMs development.

We thank Mario Trujillo for his technical assistance.

\section{References}

1. Bridges ND, Jonas RA, Mayer JE, Flanagan MF, Keane JF, Castaneda AR. Bidirectional cavopulmonary anastomosis as interim palliation for high risk Fontan candidate. Early results. Circulation. 1990;82(Suppl 4):170-6.

2. Reddy VM, Liddicoat JR, Hanley FL. Primary bidirectional superior cavopulmonary shunt in infants between 1 and 4 months of age. Ann Thorac Surg. 1995;59:1120-6.

3. Cloutier A, Ash JM, Smallhorn JF, et al. Abnormal distribution of pulmonary blood flow after the Glenn shunt or Fontan procedure: risk of development of arteriovenous fistulae. Circulation. 1985;72:471-9.

4. Chang RR, Alejos JC, Atkinson D, et al. Bubble contrast echocardiography in detecting pulmonary arteriovenous shunting in children with univentricular heart after cavopulmonary anastomosis. J Am Coll Cardiol. 1999;33:2052-8.

5. Vettukattil JJ, Slavik Z, Lamb RK, et al. Intrapulmonary arteriovenous shunting may be a universal phenomenon in patients with the superior cavopulmonary anastomosis: a radionuclide study. Heart. 2000;83: 425-8.

6. Duncan BW, Kneebone JM, Chi EY, et al. A detailed histological analysis of pulmonary arteriovenous malformations in children with cyanotic congenital heart disease. J Thorac Cardiovasc Surg. 1999; 117:931-8.

7. Srivastava D, Preminger T, Lock JE, et al. Hepatic venous blood and the development of pulmonary arteriovenous malformations in congenital heart disease. Circulation. 1995;92:1217-22.

8. Malhotra SP, Reddy VM, Thelitz S, et al. The role of oxidative stress in the development of pulmonary arteriovenous malformations after cavopulmonary anastomosis. J Thorac Cardiovasc Surg. 2002;124: 479-85.

9. Matsumoto K, Nakamura T. Emerging multipotent aspects of hepatocyte growth factor. J Biochem (Tokyo). 1996;119:591-600.

10. Ohmichi H, Koshimizu U, Matsumoto K, Nakamura T. Hepatocyte growth factor (HGF) acts as a mesenchyme-derived morphogenic factor during fetal lung development. Development. 1998;125:131524.

11. Yanagita K, Nagaike M, Ishibashi H, Niho Y, Matsumoto K, Nakamura T. Lung may have an endocrine function producing hepatocyte growth factor in response to injury of distal organs. Biochem Biophys Res Commun. 1992;182:802-9.

12. Nakamura Y, Morishita R, Higaki J, et al. Expression of local hepa- 
tocyte growth factor system in vascular tissues. Biochem Biophys Res Commun. 1995;215:483-8.

13. Yamamoto K, Morishita R, Hayashi S, et al. Contribution of Bcl-2, but not Bcl-xL and Bax, to antiapoptotic actions of hepatocyte growth factor in hypoxia-conditioned human endothelial cells. Hypertension. 2001;37:1341-8.

14. Riemer RK, Buscher C, Bansal RK, Black SM, He Y, Natuzzi E. Increased expression of nitric oxide synthase in the myometrium of the pregnant rat uterus. Am J Physiol. 1997;272:E1008-15.

15. Bernstein HS, Brook MM, Silverman NH, Bristow J. Development of pulmonary arteriovenous fistulae in children after cavopulmonary shunt. Circulation. 1995;92:309-14.

16. Liu Y, Tolbert EM, Lin L, et al. Up-regulation of hepatocyte growth factor receptor: an amplification and targeting mechanism for hepatocyte growth factor action in acute renal failure. Kidney Int. 1999;55: $442-53$.

17. Himeno W, Akagi T, Furui J, et al. Increased angiogenic growth factor in cyanotic congenital heart disease. Pediatr Cardiol. 2003;24:127-32.

18. To Y, Dohi M, Matsumoto K, et al. A two-way interaction between hepatocyte growth factor and interleukin-6 in tissue invasion of lung cancer line. Am J Respir Cell Mol Biol. 2002;27:220-6.

19. Ding S, Merkulova-Rainon T, Han ZC, Tobelem G. HGF receptor up-regulation contributes to the angiogenic phenotype of human endothelial cells and promotes angiogenesis in vitro. Blood. 2003;101: 4816-22.

20. Haddad JJ, Choudhary KK, Land SC. The ex vivo differential expression of apoptosis signaling cofactors in the developing perinatal lung: essential role of oxygenation during the transition from placental to pulmonary-based respiration. Biochem Biophys Res Commun. 2001; 281:311-6.

21. Hayashi S, Morishita R, Higaki J, et al. Autocrine-paracrine effects of overexpression of hepatocyte growth factor gene on growth of endothelial cells. Biochem Biophys Res Commun. 1996;220:539-45.

22. Wajih N, Sane DC. Angiostatin selectively inhibits signaling by hepatocyte growth factor in endothelial and smooth muscle cells. Blood. 2003;101:1857-63.

23. Nakamura Y, Morishita R, Higaki J, et al. Hepatocyte growth factor is a novel member of the endothelium-specific growth factors: additive stimulatory effect of hepatocyte growth factor with basic fibroblast growth factor but not with vascular endothelial growth factor. $J \mathrm{Hy}$ pertens. 1996;14:1067-72.

24. Ikai A, Shirai M, Nishimura K, et al. Hypoxic pulmonary vasoconstriction disappears in a rabbit model of cavopulmonary shunt. J Thorac Cardiovasc Surg. (in press).

25. Knight WB, Mee RBB. Acura for pulmonary arteriovenous fistulas? Ann Thorac Surg. 1995;59:999-1001.

26. Shah MJ, Rychik J, Fogel MA, Murphy JD, Jacobs ML. Pulmonary AV malformations after superior cavopulmonary connection: resolution after inclusion of hepatic veins in the pulmonary circulation. Ann Thorac Surg. 1997;63:960-3.

27. Laberge JM, Brandt ML, Lebecque P, et al. Reversal of cirrhosis related pulmonary shunting in two children by orthotopic liver transplantation. Transplantation. 1992;53:1135-8.

\section{Discussion}

Dr Steven F. Bolling (Ann Arbor, Mich). What do you consider your next step? Are there either blockers of hepatocyte growth factor or receptor blockers of the c-Met that can be used in the system?

Dr Ikai. Maybe we can use some HGF receptor blocker for the total animal model studied. But still they are contrary to expression of c-Met, and the c-Met expression promotes, releases some cytokine interleukin-6 or tumor growth factor-beta tumor necrosis factor out of something. So still it's very difficult to understand what kind of mechanism is involved.

Dr Hikaru Matsuda (Osaka, Japan). This is a very nice study and an interesting issue in this field of congenital heart disease. I guess this is a very big step to go into more and to focus on what is really the hepatic factor causing or preventing the AV malformation in the lung.

You show some included expression of HGF or c-Met receptor in the lung, in the cavopulmonary shunt lung. And also your previous data showed by contrast echocardiography that this model has developed arteriovenous (AV) malformation. My question is whether or not you have direct evidence of this change in expression in the place where you are histologically indicating the AV malformation in that specimen?

Dr Ikai. Actually, we didn't figure out a specific place of the PAVM expression in the lung. But in all of the animals after the 8th week of surgery, we have confirmed the PAVM development by contrast echocardiography. So these lambs should have the PAVM with some pathological changes in the lung. And meanwhile through the development of PAVM, this lung should have some change related to that, including the hepatic vein. But we don't have any pathological evidence of the PAVM in this model.

Dr Matsuda. If we can get the specimen from the patient who developed AV malformation, in that specimen we could examine what kind of growth factor and other things have developed.

I think that the HGF and Bcl-2 can be modulated or increased or depressed in various situations, including ischemia and other insult, as you have included in your model. We have been studying with another way to test whether the HGF can promote angiogenesis in the setting of very hypoplastic pulmonary circulation, such as pulmonary atresia or the congenital situation.

And so far we have shown an increase in capillary density, or number of the capillaries in the endothelial cells, but so far such angiogenesis seems to be in a normal pattern, not abnormal angiogenesis suggesting the AV malformation. But we are not sure that angiogenesis caused by HGF is not related to the malformation or just developing normal endothelial or capillary development. Can you comment on that, the HGF effect on such a situation?

Dr Ikai. Probably our experiment studied different parts. You give the human recombinant HGF for the mouse or rat. I know that result. But our model is using the very young growing lamb and this HGF and c-Met expression spontaneously occurs in the lung. Probably this expression relates to lung development and is still apparent after these lung microvasculatures are developing. Maybe this one relates to the PAVM development. So it's a big difference using the human recombinant for the angiogenesis spontaneously relating the expression of c-Met and HGF in the lung. 